\title{
Adjunct effect of music therapy on cognition in Alzheimer's disease in Taiwan: a pilot study
}

This article was published in the following Dove Press journal:

Neuropsychiatric Disease and Treatment

4 February 2015

Number of times this article has been viewed

\author{
Chien-Hsun $\mathrm{Li}^{1-3}$ \\ Ching-Kuan Liu'2,3 \\ Yuan-Han Yang ${ }^{2-4}$ \\ Mei-Chuan Chou ${ }^{2,4}$ \\ Chun-Hung Chen ${ }^{2}$ \\ Chiou-Lian Lai ${ }^{2,3}$
}

'Department of Neurology, Kaohsiung Municipal Hsiao-Kang Hospital, Kaohsiung Medical University, Kaohsiung, Taiwan; ${ }^{2}$ Department of Neurology, Kaohsiung Medical University Hospital, Kaohsiung, Taiwan: ${ }^{3}$ Department of and Master's Program in Neurology, School of Medicine, College of Medicine, Kaohsiung Medical University, Kaohsiung, Taiwan; ${ }^{4}$ Department of Neurology, Kaohsiung Municipal Ta-Tung Hospital, Kaohsiung, Taiwan
Correspondence: Chiou-Lian Lai Kaohsiung Medical University, No 100, Tzyou Ist Road, Kaohsiung 80754, Taiwan Tel +8867312 । I0। Ext 6559

Fax+88673162158

Email cllai@kmu.edu.tw
Purpose: Music therapy (MT) reviews have found beneficial effects on behaviors and social interaction in Alzheimer's disease (AD) but inconsistent effects on cognition. The purpose of the study was to evaluate the adjunct effect of long-term and home-based MT in AD patients under pharmacological treatment.

Patients and methods: Mild AD cases (clinical dementia rating $=0.5 \sim 1$ ) were consecutively recruited and voluntarily separated into an MT group or control group (CG) for 6 months. Outcome assessments included Cognitive Abilities Screening Instrument (CASI), CASI-estimated minimental state examination, clinical dementia rating with sum of box scores, and neuropsychiatric inventory. The MT interventions were Mozart's Sonata (KV 448) and Pachelbel's Canon, listening with headphones for 30 minutes daily in the morning and before sleep, respectively.

Results: Forty-one cases (MT versus CG number $=20$ versus 21) were analyzed. Adjusted differences of CASI-estimated mini-mental state examination and CASI after 6 months in the MT group were slightly less decreased than the CG without statistical significance. In further analysis of cognitive domains of CASI, the adjusted difference of abstraction domain in the MT group was significantly better than the CG.

Conclusion: Although there were no apparent additional benefits of this MT on the global cognition and daily functioning in mild AD patients, it confirms the adjunct cognition effect on the abstraction. This MT contributes to the supplementary treatment of AD.

Keywords: abstraction, behavioral and psychological symptoms of dementia, cross-cultural effect, Mozart effect, non-pharmacological intervention, supplementary treatment

\section{Introduction}

Alzheimer's disease (AD), known as one of the degenerative brain diseases, is the most common form of dementia, a clinical syndrome characterized by the progressive cognitive decline with behavioral and psychological disturbance. Pharmacological interventions (eg, acetylcholinesterase inhibitor, AchEI) are available currently but have limited ability to treat many of the syndrome's features and result in common side effects. ${ }^{1}$ Many non-pharmacological interventions have been developed to stimulate abilities, slow cognitive deterioration, reduce problematic behaviors, and improve quality of life associated with dementia. ${ }^{1}$ Music therapy (MT) is one type of non-pharmacological intervention, which is defined by the World Federation of Music Therapy as the professional use of music and its elements (sound, rhythm, melody, and harmony) as an intervention in medical, educational, and everyday environments with individuals, groups, families, or communities who seek to optimize their quality of life and improve their physical, social, communicative, emotional, intellectual, and spiritual health and wellbeing. Research, practice, education, and clinical training in MT are based on professional standards according to cultural, social, and political contexts. ${ }^{2}$ 
The most widely used method in the context of dementia is receptive relaxation MT. Tabloski et al showed that calming music such as Pachelbel's Canon was an effective, non-pharmacologic strategy that nurses and other caregivers might use to reduce agitated behavior in cognitively impaired nursing home residents. ${ }^{3}$ As early as 1998, Johnson et al reported that an individual with $\mathrm{AD}$ had an enhancement in spatial-temporal reasoning after listening to a Mozart piano sonata in a twin study. ${ }^{4}$ Some reviews have focused on the use of music for people with dementia and found beneficial effects on their behaviors and social interaction but inconsistent effects on their cognition. ${ }^{1,5}$

The first movement "allegro con spirito" of Mozart's Sonata for Two Pianos (KV 448) is referred to as the "Mozart effect" on intelligence enhancement and considered to be associated with the organization of the cortical firing pattern for spatial-temporal processes, although some researchers have refuted the findings. ${ }^{6-8}$ On the other hand, Pachelbel's Canon music was considered as having a calming and anxiolytic effect on emotions and somewhat on cognition with influences on the limbic system and parasympathetic nerve system. ${ }^{3,9,10}$ Both were well-known and studied classic music pieces for MT in the western world. Another interesting issue is that an individual's response to a particular piece of music can also mediate the effect of the music, which depends on some factors such as familiarity and preference. ${ }^{11}$ Therefore, it needs to be reappraised whether the musical effect of Mozart's piano sonata and Pachelbel's Canon is similar or not under the influence of different culture-related unfamiliarities in eastern countries, such as Taiwan. In order to evaluate the adjunct effect of medium- to long-term and home-based MT on the cognition and behavior of people with $\mathrm{AD}$ under usual pharmacological treatment, we conducted this case-control study for 6 months $(6 \mathrm{M})$.

\section{Materials and methods Design and participants}

A quasi-experimental trial design was used. The AD patients, eligible to inclusion criteria and under the consent of both patient and family, were separated into the study (MT) or control groups according to their voluntary acceptance of adjunct MT or not. The inclusion criteria for participants were: 1) AD patients, clinically diagnosed according to Diagnostic and Statistical Manual of Mental Disorders IV criteria, were in mild stage (clinical dementia rating scale, CDR $=0.5 \sim 1)^{12,13}$ under an AchEI treatment for at least 3 months in an out-patient department of neurology of a medical center hospital; 2) without severe hearing loss; 3) without major psychiatric disorders; 4) without unstable illness status; and 5) family or caregiver could assist the patient to receive the MT and follow the study protocol.

This study was approved by an ethics committee of the Institutional Review Board in the Kaohsiung Medical University Hospital. All participants and their families signed an informed consent form for participation in the study.

We consecutively recruited 87 participants from April 2008 to May 2010. Fifty-two cases participated in the study (MT) group and 35 cases were in the control group. After the $6 \mathrm{M}$ MT and follow-up course, there were 20 remaining cases in the MT group and 21 cases in the control group. Thus, 41 cases completed the study course for $6 \mathrm{M}$ and were then evaluated.

\section{Outcome assessment}

All of the participants underwent a clinical evaluation and neuropsychological assessments on baseline and after the $6 \mathrm{M}$ study course. These neuropsychological assessments of clinical MT effect included the Cognitive Abilities Screening Instrument (CASI), ${ }^{14} \mathrm{CDR}$ with sum of box scores (CDR$\mathrm{SB})^{12,13}$ and neuropsychiatric inventory (NPI, 12 terms). ${ }^{15}$ The CASI evaluated nine or ten cognitive domains: long-term memory, short-term memory (STM), orientation, attention (ATT), concentration, category fluency (FLU), language, reasoning (combination of abstraction [ABS] and judgment), and visual construction. Its total score was 100 with higher scores indicating better cognitive ability. Some of the CASI items were comparable to items used in the mini-mental state examination (MMSE).${ }^{16}$ Therefore, a CASI-estimated MMSE score, or MMSE-CE was also obtained.

The CDR is a five point scale used to characterize six domains of cognitive and functional performance applicable to $\mathrm{AD}$ and related dementias: memory, orientation, judgment and problem solving, community affairs, home and hobbies, and personal care. The global CDR scale is derived from the scores in each of the six categories ("box scores") for the assessment of the clinical severity. We also used the total sum of six box scores, CDR-SBs to evaluate the therapeutic effect of MT that might influence the dementia progression with daily functioning. The NPI total scores of 12 items were used to assess the behavioral and psychological symptoms of dementia (BPSD) with changes in patients after the MT for $6 \mathrm{M}$.

\section{MT intervention}

We used the receptive, listening-based MT method for convenient administration among the home-based patients. The intervention modes of MT consisted of the excerpts of Mozart's Sonata for Two Pianos in D major (KV 448) $)^{17}$ 
and Pachelbel's Canon in D major for Violins. ${ }^{18}$ We repetitively recorded the first movement of Mozart's sonata onto a portable digital music player for 30 minutes, as well as Pachelbel's Canon for the second track. The MT group patients and caregivers were first instructed to use the music player with music playing himself/herself at home. The piece of Mozart's sonata was listened to through headphones for at least 30 minutes daily in the morning and Pachelbel's Canon was listened to before sleep. The MT group patients and caregivers were also instructed to record the listening time and response in a music log daily. The research assistant irregularly and review the music log monthly.

\section{Statistical analysis} groups were compared by $\chi^{2}$ test. Independent two sample Student's $t$-tests were used to compare the continuous variables (age and education level, etc) and differences in the two groups in pre-test and post-test assessments. Further, the analysis of covariance (ANCOVA) was used to compare the differences between groups while adjusting for sex, age, ments. Statistical significance was defined as a $P$-value less than 0.05 . The statistical analysis was performed with SPSS software v19.0 (IBM Corporation, Armonk, NY, USA).

\section{Results}

Finally, a total of 41 cases who completed the $6 \mathrm{M}$ course was analyzed in our study; 20 cases were in the study (MT) group and 21 cases were in the control group. Females outnumbered males in both groups. The mean age, education level, baseline MMSE-CE, CASI and NPI, including depression symptom scores of both groups were similar (Table 1). Only the baseline CDR-SB scores were significantly

Table I Demographic and clinical characteristics of the two groups

\begin{tabular}{|c|c|c|c|}
\hline & $\begin{array}{l}\text { MT group } \\
(n=20)\end{array}$ & $\begin{array}{l}\text { Control group } \\
(n=2 I)\end{array}$ & $P$-value \\
\hline Sex (female/male) ${ }^{\mathrm{a}}$ & $15 / 5$ & $13 / 8$ & 0.368 \\
\hline Age $(y \pm S D)^{b}$ & $76.7 \pm 8.45$ & $80.8 \pm 5.94$ & 0.082 \\
\hline Education level (years) & $6.7 \pm 4.77$ & $5.8 \pm 4.17$ & 0.506 \\
\hline MMSE-CE (baseline, $M \pm S D$ ) & $17.7 \pm 5.98$ & $14.7 \pm 3.35$ & 0.062 \\
\hline CASI & $59.0 \pm 18.77$ & $51.1 \pm 13.55$ & 0.129 \\
\hline CDR-SB & $5.4 \pm 1.67$ & $6.9 \pm 1.59$ & $0.004 *$ \\
\hline NPI & $7.4 \pm 13.17$ & $9.1 \pm 12.75$ & 0.660 \\
\hline
\end{tabular}

Notes: ${ }^{a} \chi^{2}$ test; ' two sample Student's $t$-test for continuous variables. $* P<0.05$. Abbreviations: CASI, Cognitive Abilities Screening Instrument; CDR-SB, clinical dementia rating scale with sum of box scores; M, mean; MMSE-CE, CASI-estimated mini-mental state examination; MT, music therapy; NPI, neuropsychiatric inventory; $\mathrm{SD}$, standard deviation; $y$, years. would monitor the participants' compliance by telephone

The distributions of categorical variable (sex) between the two education level, NPI, and corresponding baseline measure-

different between the two groups (MT versus [vs] control group $=5.4 \pm 1.67$ vs $6.9 \pm 1.59, P=0.004)$ (Table 1$)$.

After comparing group data of the $6 \mathrm{M}$ period, the $6 \mathrm{M}$ differences of MMSE-CE and CASI in the MT group were $-0.9 \pm 3.09$ and $-0.9 \pm 6.62$ vs $-0.6 \pm 2.73$ and $-2.1 \pm 7.84$ in the control group, both without statistical significance between groups. The $6 \mathrm{M}$ differences of CDR-SB and NPI between groups were also without significant difference $(0.6 \pm 1.97$ and $1.5 \pm 16.39$ vs $0.6 \pm 0.63$ and $2.9 \pm 10.69)$ (Table 2). Further 6M differences of MMSE-CE, CASI, CDR-SB, and NPI were adjusted for covariates of sex, age, education level, NPI and corresponding baseline measurements, using ANCOVA. These adjusted differences of MMSE-CE and CASI after $6 \mathrm{M}$ in the MT group $(-0.6 \pm 0.70$ [standard error] and $0.1 \pm 1.87)$ were slightly less decreased than those in the control group $(-0.8 \pm 0.68$ and $-3.0 \pm 1.82)$. However, they did not reach statistical significance $(P=0.827$ and 0.295 , respectively) (Table 2). The adjusted differences of CDR-SB and NPI in the MT group were less increased than those in the control group without statistical significance $(0.5 \pm 0.36$ vs $0.7 \pm 0.35$ and $0.4 \pm 2.94$ vs $3.9 \pm 2.85, P=0.718$ and 0.431 , respectively) (Table 2).

In further analyzing and comparing the cognitive domains of CASI between groups, the $6 \mathrm{M}$ difference in the ABS domain in the MT group was significantly better than that of the control group $(0.10 \pm 1.25$ vs $-0.90 \pm 1.45, P=0.023$; adjusted difference $=0.10 \pm 0.29$ vs $-0.90 \pm 0.28, P=0.024$ ).

Table 2 Comparison of cognitive and neuropsychiatric assessments between MT and control groups

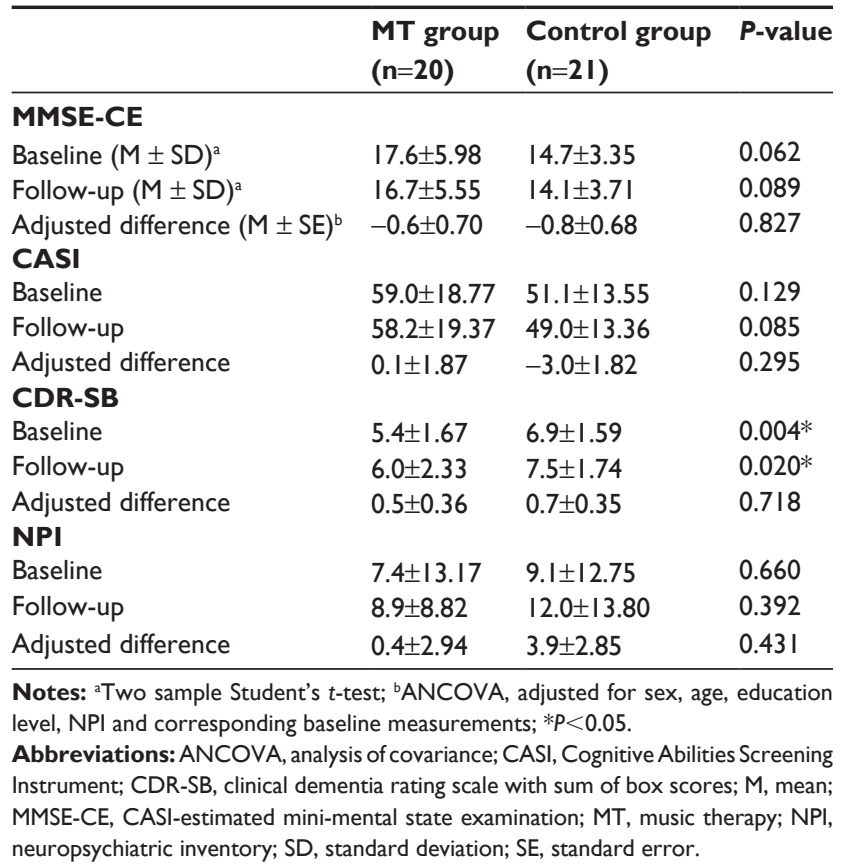

Neuropsychiatric Disease and Treatment 20I5:I I

submit your manuscript $\mid$ www.dovepress.com 
The other cognitive domains were not significantly different between groups after the $6 \mathrm{M}$ study (Table 3 ). However, the $6 \mathrm{M}$ difference in the domain of STM had a trend toward being better in the MT group than in the control group $(0.34 \pm 1.91$ vs $-0.56 \pm 1.56, P=0.107$; adjusted difference $=0.37 \pm 0.43$ vs $-0.60 \pm 0.42, P=0.135)$.

\section{Discussion}

In the present study, a significant improvement in ABS was noted in the MT group, although the other cognitive domains did not show a difference between the two groups. Abstracting similarities between pairs of items are administered in CASI to provide a profile of abstract function. Abstract thinking is the ability to deal with concepts. It is one part of reasoning and high executive functions, which are activated and integrated in the frontal and temporal lobes. ${ }^{19}$ This result is consistent with the finding of Johnson et al's study in 1998, though using a different test, it also supported the Mozart effect in AD patients with different cultures. He presumed that an $\mathrm{AD}$ patient had enhancement of spatial-temporal

Table 3 Comparison of CASI domains between MT and control groups

\begin{tabular}{|c|c|c|c|}
\hline Cognitive domains & $\begin{array}{l}\text { MT group } \\
(n=20)\end{array}$ & $\begin{array}{l}\text { Control group } \\
(n=21)\end{array}$ & $P$-value \\
\hline LTM baseline $(M \pm S D)^{a}$ & $8.25 \pm 2.31$ & $8.33 \pm 2.15$ & 0.906 \\
\hline Adjusted difference $(M \pm S E)^{b}$ & $0.07 \pm 0.35$ & $-0.2 I \pm 0.34$ & 0.585 \\
\hline ORT baseline & $10.95 \pm 4.38$ & $8.38 \pm 3.34$ & $0.04 I^{*}$ \\
\hline Adjusted difference & $-0.66 \pm 0.73$ & $-0.57 \pm 0.7 \mid$ & 0.936 \\
\hline ATT baseline & $6.25 \pm 1.62$ & $5.38 \pm 1.94$ & 0.128 \\
\hline Adjusted difference & $-0.32 \pm 0.39$ & $-0.22 \pm 0.38$ & 0.856 \\
\hline CCT baseline & $4.45 \pm 3.78$ & $3.62 \pm 2.91$ & 0.433 \\
\hline Adjusted difference & $0.66 \pm 0.47$ & $0.09 \pm 0.46$ & 0.415 \\
\hline STM baseline & $3.74 \pm 3.07$ & $2.18 \pm 2.40$ & 0.077 \\
\hline Adjusted difference & $0.37 \pm 0.43$ & $-0.60 \pm 0.42$ & 0.135 \\
\hline FLU baseline & $3.70 \pm 2.27$ & $3.33 \pm 2.39$ & 0.618 \\
\hline Adjusted difference & $-0.39 \pm 0.44$ & $-0.54 \pm 0.43$ & 0.603 \\
\hline LAN baseline & $7.29 \pm 2.57$ & $6.8 \mathrm{I} \pm 2.10$ & 0.520 \\
\hline Adjusted difference & $-0.18 \pm 0.28$ & $0.04 \pm 0.27$ & 0.594 \\
\hline ABS baseline & $2.60 \pm \mathrm{I} .57$ & $2.57 \pm 1.75$ & 0.956 \\
\hline Adjusted difference & $0.10 \pm 0.29$ & $-0.90 \pm 0.28$ & $0.024 *$ \\
\hline JUD baseline & $4.55 \pm 1.19$ & $4.05 \pm 1.12$ & 0.171 \\
\hline Adjusted difference & $-0.06 \pm 0.24$ & $0.01 \pm 0.24$ & 0.857 \\
\hline REA baseline & $7.15 \pm 2.32$ & $6.62 \pm 2.38$ & 0.474 \\
\hline Adjusted difference & $-0.02 \pm 0.37$ & $-0.87 \pm 0.36$ & 0.111 \\
\hline VC baseline & $6.70 \pm 3.23$ & $6.05 \pm 2.87$ & 0.498 \\
\hline Adjusted difference & $0.55 \pm 0.50$ & $-0.38 \pm 0.49$ & 0.213 \\
\hline
\end{tabular}

Notes: ${ }^{\mathrm{T} T w o}$ sample $t$-test; ${ }^{\mathrm{b}} \mathrm{ANCOVA}$, adjusted for sex, age, education level, NPI and corresponding baseline measurements; ${ }^{*} P<0.05$.

Abbreviations: ABS, abstraction; ANCOVA, analysis of covariance; ATT, attention; CASI, Cognitive Abilities Screening Instrument; CCT, concentration; FLU, category fluency; JUD, judgment; LAN, language; LTM, long-term memory; M, mean; MT, music therapy; NPI, neuropsychiatric inventory; ORT, orientation; REA, reasoning (abstraction + judgment); SD, standard deviation; SE, standard error; STM, shortterm memory; VC, visual construction. reasoning after listening to Mozart's sonata under the assessment of the Stanford-Binet Intelligence Scale. ${ }^{4}$ The possible mechanisms of the Mozart effect or music enhancement are that cortical activation is directly affected by general arousal with ATT, thus improving cognitive task performance. ${ }^{20,21}$ The long-term mechanisms may be related to the increase of cerebral blood flow and cortex circuit activation in the frontal and temporal lobes, ${ }^{22,23}$ as well as several neurotransmitters (eg, dopamine), neuropeptides (endorphins and endocannabinoids), and other biochemical mediators (eg, nitric oxide) that play some roles in the perceptual and emotional processing of music. ${ }^{24}$ In addition, there was potential improvement of STM in the MT group yet without statistical significance. There is the promise of significant improvement in the cognitive domain after longer or rigorous MT intervention in future research or in a clinical setting.

Several studies have demonstrated that MT provides some beneficial effects for AD patients in terms of cognitive performance, including spatial-temporal reasoning, language content and fluency, ATT, and autobiographical memory recall under the assessment of variant neuropsychological tests. ${ }^{4,25-27}$ However, Vink et al had updated their rigorous Cochrane review of MT for dementia in 2010 and still came to no useful conclusions. In terms of inconsistent results, most studies were too small with methodological limitations and concerned short-term therapy effects (intervention periods less than 4 months) in institutionalized elders. ${ }^{1}$ In our study, the MMSE-CE did not show significant differences between groups. Because of the brief and limited characteristics of the MMSE, it may be difficult to detect the intra-individual changes in cognition during the relative short period of dementia progression. ${ }^{28}$

The CASI and CDR-SB were concomitantly used to evaluate more comprehensive cognition with daily functioning. Moreover, the CASI total score favorably compares with the Alzheimer's Disease Assessment Scale (ADAS$\operatorname{cog}$ ), the most widely used to assess the effect of drug treatment for $\mathrm{AD}$, in both score range and retest reliability. ${ }^{29} \mathrm{It}$ is a pity that both CASI and CDR-SB also had no significant improvement in the global cognition and daily functioning of an MT group after comparison with a control group. However, the MT group had slightly less deterioration than the control group in the assessment of CASI. In other words, the adjunct MT might have potentially synergetic benefits in slowing the deteriorating process of $\mathrm{AD}$ with AchEI treatment.

In respect of behavioral and psychological effects after $6 \mathrm{M} \mathrm{MT}$, there was no significant alleviation under the NPI assessment in the MT group. However, the MT group 
revealed slightly less exacerbation of BPSD than the control group after adjusting for cognitive status. Somehow the MT might have the efficacy of stabilization of BPSD in the longterm care of $\mathrm{AD}$. In order to obtain the deeper response of this MT on behavior and emotion, further detailed analysis of NPI items should be considered.

There are some particular advances in our study. First, the well-verified and available neuropsychological tests (CASI and CDR-SB) were used clinically in the assessment of cognition and daily functioning. It is possibly reproducible and comparable with the cognitive effects of other MT modes or types under the same neuropsychological tests. Secondly, the study included only mildly affected AD patients under the stationary treatment of AchEI over 3 months. It might avoid the known bias of cognitive effect of medication in the MT group after comparison with the control group. Thirdly, it was convenient and simple to administer the MT with the use of a portable digital music player with headphones in longterm community patients. It is not limited to institutional or therapeutic groups or necessarily to administration and performance by a well-qualified music therapist. Clinical physicians may easily approach this MT type as a supplementary therapy in situations with less available music therapists in most clinical settings. Moreover, families or caregivers are also actively assisting in the procedure and play an important role in the treatment course. Finally, in spite of different cultural backgrounds, this MT still had partial cross-cultural effects on cognition. The participants' response to MT was enhanced when the music was repeated to enhance familiarity over the long term. ${ }^{11}$ Music intervention might be suggested in dementia even in people with an eastern cultural background or those who are unfamiliar with MT.

One of the limitations of our study is the relatively high drop-out ratio (61.5\% vs $40.0 \%$ between groups), which can be expected in vulnerable AD patients and a long-term duration study. The major reasons for drop-out in the MT group were due to the dullish sensation of limited music modes and impatience with rigorous study rules in the first 2 months. Therefore, the final subject number of each group is relatively small. The results of behavioral measures might be underpowered. Another weak point of this study is the quasi-experimental design without randomized and placebo-controlled groups due to ethical concerns and clinical outpatient investigations. It might have the bias of expected psychological and placebo effects of MT intervention in the MT group. Whether we would be more intent to treat with better effect is worthy of future investigation. The other limitation is the insufficient information on medications in each participant. Some medications might influence the mental functioning and have a confounding effect on cognition and BPSD. Further better and more rigorous methodological designs are welcomed to obtain the pure cognition effect of MT with increased study evidence and more research resources.

\section{Conclusion}

Although there were no apparent additional benefits of this MT mode on global cognition and daily functioning in mild $\mathrm{AD}$ patients for $6 \mathrm{M}$, it confirms the adjunct cognition effect of MT on ABS and potential STM. Furthermore, more rigorous methodological designs with sensitive psychometric instruments are welcomed to ascertain the promising efficacy of MT on global cognition and BPSD. This MT mode, which is easily applied and includes the caregivers, contributes to the supplementary treatment of dementia syndrome in AD.

\section{Acknowledgments}

The authors thank the Statistical Analysis Laboratory, Department of Medical Research, Kaohsiung Medical University Hospital, Kaohsiung Medical University for their help. This study was supported by a grant from the Kaohsiung Medical University Hospital (KMUH966G40).

\section{Author contributions}

All authors contributed toward data analysis, drafting and revising the paper and agree to be accountable for all aspects of the work.

\section{Disclosure}

The authors report no conflicts of interest in this work.

\section{References}

1. Vink AC, Bruinsma MS, Scholten RJ. Music therapy for people with dementia. Cochrane Database Syst Rev. 2011(3):CD003477.

2. World Federation of Music Therapy. FAQ MUSIC THERAPY. 2011 [cited 2012 May 23]. Available from: http://www.wfmt.info/WFMT. Accessed June 6, 2014.

3. Tabloski PA, Mckinnon-Howe L, Remington R. Effects of calming music on the level of agitation in cognitively impaired nursing home residents. Am J Alzheimers Dis Other Demen. 1995;10(1): $10-15$.

4. Johnson JK, Cotman CW, Tasaki CS, Shaw GL. Enhancement of spatialtemporal reasoning after a Mozart listening condition in Alzheimer's disease: a case study. Neuro Res. 1998;20(8):666-672.

5. Sung HC, Chang AM. Use of preferred music to decrease agitated behaviours in older people with dementia: a review of the literature. J Clin Nurs. 2005;14(9):1133-1140.

6. Rauscher FH, Shaw GL, Ky KN. Music and spatial task performance. Nature. 1993;365(6447):611.

7. Rauscher FH, Shaw GL. Key components of the Mozart effect. Percept Mot Skill. 1998;86(3 Pt 1):835-841. 
8. Steele KM, Dalla Bella S, Peretz I, et al. Prelude or requiem for the Mozart effect? Nature. 1999;400:827.

9. Cash AH, el-Mallakh RS, Chamberlain K, Bratton JZ, Li R. Structure of music may influence cognition. Percept Mot Skilsl. 1997;84(1):66.

10. Knight WEJ, Rickard NS. Relaxing music prevents stress-induced increases in subjective anxiety, systolic blood pressure, and heart rate in healthy males and females. J Music Ther. 2001;38(4):254-272.

11. Abeles HF, Chung JW. Responses to music. In: Hodges DA editor. Handbook of music psychology. San Antonio: University of Texas; 1996:285-342.

12. Morris JC. The clinical dementia rating (CDR): Current version and scoring rules. Neurology. 1993;43(11):2412-2414.

13. Lin KN, Liu HC. [Clinical Dementia Rating (CDR), Chinese Version]. Acta Neurol Taiwan. 2003;12(3):155-165. Chinese.

14. Lin KN, Wang PN, Liu CY, Chen WT, Lee YC, Liu HC. Cutoff scores of the Cognitive Abilities Screening Instrument, Chinese version in screening of dementia. Dement Geriatr Cogn Disord. 2002;14(4):176-182.

15. Cummings JL. The Neuropsychiatric Inventory: assessing psychopathology in dementia patients. Neurology. 1997;48(Suppl 6):S10-S16.

16. Folstein M, Folstein S, Mchugh P. "Mini-mental state": a practical method for grading the cognitive state of patients for the clinician. J Psychiatr Res. 1975;12(3):189-198.

17. Mozart. Sonata in D for Two Pianos, KV 448: I. Allegro con spirito. [cited 2012 May 23]. Available from: http://www.mozart-archiv.de. Accessed May 23, 2012.

18. Pachelbel. CANON AND GIGUE. In MIND OF NATURE DISC 2. HOMERUN PRODUCTION (Taiwan Music No 0958), 2007.

19. Wang J, Conder JA, Blitzer DN, Shinkareva SV. Neural representation of abstract and concrete concepts: A meta-analysis of neuroimaging studies. Hum Brain Mapp. 2010;31(10):1459-1468.
20. Thompson WF, Schellenberg EG, Husain G. Arousal, mood, and the Mozart effect. Psychol Sci. 2001;12(3):248-251.

21. Jausovec N, Habe K. The "Mozart effect": an electroencephalographic analysis employing the methods of induced event-related desynchronization/synchronization and event-related coherence. Brain Topogr. 2003;16(2):73-84.

22. Blood AJ, Zatorre RJ, Bermudez P, Evans AC. Emotional responses to pleasant and unpleasant music correlate with activity in paralimbic brain regions. Nat Neurosci. 1999;2(4):382-387.

23. Koelsch S, Fritz T, Cramon DY, Muller K, Friederici AD. Investigating emotion with music: an fMRI study. Hum Brain Mapp. 2006;27(3): 239-250.

24. Blood AJ, Zatorre RJ. Intensely pleasurable responses to music correlate with activity in brain regions implicated in reward and emotion. Proc Natl Acad Sci U S A. 2001;98(20):11818-11823.

25. Brotons M, Koger SM. The impact of music therapy on language functioning in dementia. J Music Ther. 2000;37(3):183-195.

26. Gregory D. Music Listening for Maintaining Attention of Older Adults with Cognitive Impairments. J Music Ther. 2002;39(4):244-264.

27. Irish $\mathrm{M}$, Cunningham $\mathrm{CJ}$, Walsh JB, et al. Investigating the enhancing effect of music on autobiographical memory in mild Alzheimer's disease. Dement Geriatr Cogn Disord. 2006;22(1):108-120.

28. Liu HC, Teng EL, Lin KN, et al. Performance on the cognitive abilities screening instrument at different stages of Alzheimer's disease. Dement Geriatr Cogn Disord. 2002;13(4):244-248.

29. Liu HC, Teng EL, Chuang YY, Lin KN, Fuh JL, Wang PN. The Alzheimer's Disease Assessment Scale: Findings from a Low-Education Population. Dement Geriatr Cogn Disord. 2002;13(1):21-26.
Neuropsychiatric Disease and Treatment

\section{Publish your work in this journal}

Neuropsychiatric Disease and Treatment is an international, peerreviewed journal of clinical therapeutics and pharmacology focusing on concise rapid reporting of clinical or pre-clinical studies on a range of neuropsychiatric and neurological disorders. This journal is indexed on PubMed Central, the 'PsycINFO' database and CAS,

\section{Dovepress}

and is the official journal of The International Neuropsychiatric Association (INA). The manuscript management system is completely online and includes a very quick and fair peer-review system, which is all easy to use. Visit http://www.dovepress.com/testimonials.php to read real quotes from published authors. 